\title{
Study on the Configuration of Cinemusic
}

\author{
Yi Ji \\ Music and Dancing Institute \\ Qujing Normal University \\ Qujing, Yunnan, China
}

\begin{abstract}
The configuration of cinemusic can not only reveal, interpret and expand the connotations of films, but also restrain or develop the imaginations of audiences by the relationship between music and frame. This paper will give an introduction to the formation and development of cinemusic, combining with specific historical materials, expound the relationship among music, audios and frame in music configuration, point out the significance of grasping and handling the relationship between music and frame. The author's opinion is that only when the relationships between music and frame are utilized appropriately can they come into effect achieve the perfect combination of music and frame, and finally generate new artistic charm.
\end{abstract}

Keywords-films; music; configuration; frame

\section{INTRODUCTION}

Chinese film industry is booming in new century. With the promotion of cinemusic configuration, study on the practice and theory of the subject has made profound achievements. The configuration of cinemusic influences the audiences' understanding and experience of the film. Those classical films are unexceptionally excelling in the configuration of cinemusic.

\section{THE FORMATION AND DEVELOPMENT OF Cinemusic CONFIGURATION}

Cinemusic configuration first appeared in silent film era. Silent film is made up frames, with no aside and music; therefore, it is called silent film. To supplement the blank of silent films and satisfy the audiences' visual and auditory demands, the pictures scheduled pianists and violinists behind the screen to accompany when playing the film. This is the primitive form of cinemusic configuration.

At the very beginning, music was not closely integrated with frames mainly because the accompaniment catered to the audiences' preference instead of real needs. They were usually popular worldly music, full of randomness and it often happened that the music was unfitting even contradictory with the frames and atmosphere. For example, some solemn frames were accompanied with noisy and lovely music, while cheerful frames with serious and sorrowful music, which resulted in the alienation of the films' visual and auditory effects and separation of audiences' aesthetic values. Therefore, psychological discord occurred to the viewers and their film appreciation was also spoiled. In order to conform the visual and auditory effects and resolve the problem, a batch of musicians were employed by the film companies to compose and compile a collection of cinemusic. Typical collections include $A$ Handbook of Cinemusic and A Collection of Cinemusic edited by an Italian composer Giuseppe Beche. In order to instruct the accompaniment to select suitable music matching the frames, the collections were divided into different sections according to their style, such as serenades, lyrical music and marches. What's more, corresponding meanings of different musical emotions were also given; for example, the emotional orientation of lyrical, scenery description and powerful music were presented on the collections. Since people had those music with specific emotional orientation, cinemusic and frames could be combined further and their relationships could be established and develop. With the development of film industry and the promotion of audiences' aesthetic values, the same musical accompaniment applying to different films could not meet the viewers' higher requirements. Therefore, apart from the extant collections, composers also created music for specific films for accompaniment. According to the historical materials, the first composer creating music for a particular film was a famous French musician, Saint-Saens, who created a music for a French film L'assassinat du duc de Guise in 1908. Then in the year of 1913, Italian composer Giuseppe Beche wrote a piece of music for the film Richard Wagner. After the 1920s, a lot of composers specialized in composing cinemusic, and they have made initiative contribution to the configuration of cinemusic, such as Eisenstein's Battleship Potemkin (written by German composer Edmund Menzel). With the development of science and technology, The Jazz Singer, made by AOL Time Warner, added to four songs and accompaniment was released to the public in 1927, which marked the birth of talkie. From then on, films upgraded from silent films, the pure visual enjoyment to talkies, the combination of visual and auditory enjoyment. The relationships among music, audio and frames could be established and make progress, and the cinemusic configuration have made huge achievements both theoretically and practically, such as The Techniques of Cinemusic coauthored by Manville and Huntley, The Aesthetics of Cinemusic written by Poland musical aesthetician Zofia, and in 1988, Sucong and American and Japanese composers cocomposed a piece of music for the film The Last Emperor, which has won the 60th Oscar for Best Original Score.

\section{The CONFIGURATION OF Cinemusic}

In the films, music and audio as auditory elements are supposed to be organically associated with visual elements, namely frames. They supplement and permeate each other. 
When the openness, abstractness and straightness of music are combined with the frames, the film viewers can deepen their imagination. Meanwhile, the content of the films becomes clearer and specified, and the audiences' understanding of the film will be restrained and the room for their imagination will be condensed.

\section{A. Relationships between Music and Frames}

According to the different sources of music, their relationships can be categorized as interior music and exterior music of frames.

1) Interior music of frames is provided by the frames: In terms of expression techniques, these music manifest as musical action, media playing and singing and dancing scenes. Thus, interior music can help the audiences get closer to the film and experience the natural and harmonious atmosphere. Especially when applied to character shaping and atmosphere rendering, interior music shows strong authenticity and reality. In the film Titanic, to show the musicians' professional ethics and lofty qualities and compare panic passengers with calm musicians, the musicians are collocated to play calm and natural music.

2) Exterior music is corresponding to interior music because it comes from out of the frames: Exterior music has strong openness, reflecting the director's objective preference and closely associated to the director's creating ideas and styles. Exterior music is to propel audiences to make full use of their imagination to objectively comment and evaluate the film. Besides, exterior music presents its creating awareness and highlights its creating styles. For instance, in Schindler's List, a slow and desperate melody is played by different instruments and applied to many frames, which reflects Jews' miseries and arouse the audiences' sympathy. Consequently, the theme of the film is presented and sublimed. In addition, interior and exterior music can frequently shift from one to another. When the authenticity of frames is ineffective, exterior music will be added to highlight the theme. Then the sentimental color becomes strong and the development of emotions is more natural and smoother.

According to the combination from of music and frame, their relationships embody three aspects: unification, confliction and juxtaposition.

- Unification of music and frame means the configuration of music and frames are unified in entirety and part; for example, the contents, the characteristic of the times and ethnic styles of the work should be consistent with cinemusic. Unification of music and frames is very common in most films because it can meet the public's visual and auditory psychological habits, strengthen the authenticity and infection of the work. Although unification can cater to the audiences' psychological expectation, innovative connotations and ideas of film are restrained and it is hard for audiences to take advantage of their objective evaluation capability.
- Confliction is inconsistency between music and frames, reflecting contrary structures and forms. With its huge contrast, goals to deepen the connotations and shock the audiences' psychology can be achieved, and intense artistic effects will be presented, such as violent frames with melodic and lyric music, lovely and cheerful contents with sorrowful music.

- Juxtaposition of music and frames refers to the alienation of images separately presented by music and frames, i.e. music and frames are neither unified nor contrary. It is a form between unification and confliction, and it is usually applied to feature films.

\section{B. Relationships between Music and Other Audios}

Audios with specific purposes are important elements in films, and they are a must for the shaping of styles, character images and environmental atmosphere; therefore, audios configuration is vital for film works. Audios consist of human voices, audios with specific purposes and music. As for film works, the word "language" refers to all sounds made by human beings' phonatory organs, including asides, dialogues, monologues and commentaries. Audios refer to sounds of wind, water and animals except from human beings and music.

The processing of music and audios has direct impact on the whole film. During the configuration, following key points should be paid more attention.

1) The proportion of configuration: Audios with specific purposes have their corresponding attributes, so they are supposed to be sorted according to their importance. To find out the leading audio and accompany with appropriate volume is helpful for emphasizing on their significance. At the same time, excessive emphasis on the music is supposed to be avoided because it may steal the show of synchronous sounds and narration. To maintain the integrity of music, although audios with specific purposes have strong and vivid effect, the music should not be interrupted too much. Therefore, in most cases, audios are placed at the beginning or end of the music.

2) The maintenance of respective integrities: When deploying music in the music, we should as much as possible sustain the integrity of audios or music because it takes a period of time for audiences to understand the music and frames. If the music or audios are interrupted frequently, the functions of music and audios and combination of visual and auditory effects will be weakened. Thereby, it is bad for the formation of montage.

\section{Relationships among music}

In film works, relationships among music are embodied in the consistency of all music and comparison of different plots. The consistency of cinemusic includes:

1) The consistency of instruments' styles and attributes: Instruments are classified by their timbres, audios and materials. For example, Western instruments include string instruments (the violin family), woodwind (flute), brass-wind instruments (tuba) and percussion (timbrel), while Chinese 
instruments contain tongue (Xiao, a vertical bamboo flute), string instruments (Zheng, a 21-or 25-stringed plucked instrument in some ways similar to the zither), percussion (chime stone, drum and gong). In deploying the music, we should pay special attention to the features of these instruments. If the previous plots are accompanied with Chinese instruments and the subsequent plots with Western instruments, the consistency and harmony of films will not be achieved.

2) The similarities of musical styles: The apparent cultural characters of music is that they possess particular styles of times and ethnic colors. In deploying music, if the melodies match the styles of frames, choosing proper music in accordance with the real plots will contribute to audiences' positive emotions and influence of films. On the contrary, provided the same melody is applied throughout the whole film or melodies representing different cultures are mixed in the film, the picture will be bland and awkward.

3) The similarities of musical mode and tonality: Mode and tonality are the key factors to feature the connotations of music, and specific ethnic customs and historical culture can always reflect their corresponding tonalities, such as traditional Chinese pentatonic mode and chorale which symbolizes medieval Western musical culture in Roman Catholic Church. Therefore, clashes of different types of modes and tonalities should be averted and modes are supposed to match the frames. In addition, distant-relationship and intimate-relationship tunes have distinct differences in their respective cohesion. Intimate-relationship tune should be adopted because it makes cohesion smoother while distantrelationship tunes will give an illusion of discontinuity.

4) General deposition: In cinemusic configuration, the first step is to grasp the fundamental key of the music, to determine the theme or echoes of the beginning and end. Specific manifestations are selecting the musical elements, such as styles of melodies, rhythm and metre, speed and strength. What's more, relationships among music are reflected by comparisons of different plots. It displays as comparisons of relief and tension of rhythm, highs and lows of musical emotion and simplicity and abundance melodies, which results in the effect of differentiation.

\section{CONCLUSION}

With the development of science and technology and the mass's ever-growing cultural needs, the configuration of cinemusic has gone through silent stage to phonic stage. People have had great achievements in theoretical and practical fields and their works are always embodies with styles of time. Thereby, it is of great necessity to differentiate the relationships among music, audios and frames, to master the approaches to deploy cinemusic, and eventually achieve the goal to propel the development of theoretical and practical cinemusic configuration.

\section{REFERENCES}

[1] Chen Bin \& Cheng Jin. Film, Television and Music [M]. Hangzhou: Zhejiang University Press, 2004.

[2] Yuan Qingfeng. A Study on the Secular Influence of Cinemusic Configuration and Transmission Effect of Chinese Film in 1937 [J]. Chinese music, 2011, (3).

[3] Zhao Xiuyan. Significant Influence of Cinemusic on Films [J]. Movie Literature, 2011, (14).

[4] Chen Dawei. Deconstruction of Cinemusic Configuration-Taking the Film Léon for Example [J]. Movie Literature, 2013, (5). 